\title{
47. DATA REPORT: PRELIMINARY GAMMA-RAY ANALYSES OF SAMPLES FROM LEG 1391
}

\author{
Willard S. Moore ${ }^{2}$ and Debra Stakes ${ }^{3}$
}

Samples from Ocean Drilling Program Leg 139 were selected for an investigation of radium isotopes by nondestructive gamma-ray analysis to determine if they contained high activities of ${ }^{226} \mathrm{Ra}$ or ${ }^{228} \mathrm{Ra}$ that could date the formation of the samples.

Preliminary shipboard inspection revealed that some of the samples contained abundant concentrations of barite, a mineral known to concentrate radium. The ratios of ${ }^{226} \mathrm{Ra} / \mathrm{Ba}$ in recently precipitated hydrothermal barites are on the order of $2000 \mathrm{dpm} / \mathrm{gm}$ (Moore and Stakes, 1990). Due to the 1600 -year half life of ${ }^{226} \mathrm{Ra}$, this ratio will decrease with time. Therefore, ${ }^{226} \mathrm{Ra} / \mathrm{Ba}$ ratios in barites could provide an index of their ages in the range 1-5 thousand years.

If we discovered samples with high ${ }^{226} \mathrm{Ra} / \mathrm{Ba}$ ratios, indicating young ages, determining the ${ }^{228} \mathrm{Ra} /{ }^{226} \mathrm{Ra}$ activity ratio would establish whether the sample was in the age range $5-30$ years because ${ }^{228} \mathrm{Ra}$ has a half life of 5.7 years. Samples with high ${ }^{226} \mathrm{Ra} / \mathrm{Ba}$ ratios and high ${ }^{228} \mathrm{Ra} /{ }^{226} \mathrm{Ra}$ activity ratios could be dated using ${ }^{228} \mathrm{Th} /{ }^{228} \mathrm{Ra}$ activity ratios because the barite should form with little ${ }^{228} \mathrm{Th}$ compared to ${ }^{228} \mathrm{Ra}$; but ${ }^{228} \mathrm{Th}$ would be produced by ${ }^{228} \mathrm{Ra}$ decay until the sample reached transient equilibrium with a ${ }^{228} \mathrm{Th} / 228 \mathrm{Ra}$ activity ratio of 1.5 (Stakes and Moore, 1991). This dating technique would be useful in the range $1-15$ years.

Each of these techniques is predicated on the enrichment of Ra isotopes relative to Th parents in barite. If the samples were more than 5 thousand years old, most of the ${ }^{226} \mathrm{Ra}$ would be due to its production by ${ }^{230} \mathrm{Th}$ in the sample, and the sample could not be dated by the excess ${ }^{226} \mathrm{Ra}$ technique. Similarly, if the expected enrichment of ${ }^{226} \mathrm{Ra}$ in barite did not occur, or if the sample did not contain significant barite, most of the ${ }^{226} \mathrm{Ra}$ in the sample could have been produced from ${ }^{230} \mathrm{Th}$ in the barite or in other phases and the sample could not be dated using these techniques.

We selected samples from the cores based on a preliminary evaluation of their barite contents. The samples were packed in counting vials and the gamma-ray activity was measured on a $\mathrm{Ge}(\mathrm{Li})$ detector. The detector was calibrated by measuring standards in approximately the same geometry; but, because the core cuttings were not crushed (nor were they dried), it was impossible to reproduce the exact geometry. Therefore, the absolute activities may be in error by as much as $\pm 25 \%$. Because most of the samples had similar geometry, relative sample-to-sample errors are probably within $\pm 15 \%$. The gamma-ray spectra were analyzed using the program HYPERMET (Phillips and Marlow, 1976). Two sigma counting errors on most samples are less than $\pm 5 \%$.

\footnotetext{
${ }^{1}$ Mottl, M.J., Davis, E.E., Fisher, A.T., and Slack, J.F. (Eds.), 1994. Proc. ODP, Sci. Results, 139: College Station, TX (Ocean Drilling Program).

${ }^{2}$ Department of Geological Sciences, University of South Carolina, Columbia, SC 29208 , U.S.A.

${ }^{3}$ Department of Geological Sciences, University of South Carolina, Columbia, SC 29208, U.S.A. (Present address: MBARI, 160 Central Avenue, Pacific Grove, CA 93950, U.S.A.)
}

The results of the samples measured are given in Table 1. None of these samples had particularly high activities of ${ }^{226} \mathrm{Ra}$. Some of them, such as $139-856 \mathrm{D}-1 \mathrm{H}-7,73 \mathrm{~cm}, 856 \mathrm{H}-17 \mathrm{R}-1,34 \mathrm{~cm}$, and $858 \mathrm{~B}-5 \mathrm{H}-$ $2,13 \mathrm{~cm}$ had higher activities than the others, but none approached the $2000 \mathrm{dpm} / \mathrm{gm}$ reported for pure, recent barites.

We conclude from this brief, preliminary examination of these samples that Ra dating of bulk samples is not possible. Either the samples are $>5000$ years old and most of the initial excess ${ }^{226} \mathrm{Ra}$ has decayed, or the abundance of barite in the samples measured was small, representing only a small fraction of the total sample. Because the activities of the parent isotopes $\left({ }^{230} \mathrm{Th}\right.$ in the case of ${ }^{226} \mathrm{Ra}$ and ${ }^{232} \mathrm{Th}$ in the case of ${ }^{228} \mathrm{Ra}$ ) are not known in these samples, activity ratios of ${ }^{228} \mathrm{Ra} /{ }^{226} \mathrm{Ra}$ or ${ }^{228} \mathrm{Th} /{ }^{228} \mathrm{Ra}$ cannot be used to calculate their ages.

The following steps would be required to obtain additional data that might enable ages to be assigned to these samples:

1. Enough barite must be separated physically or chemically so that it can be measured as a pure phase.

2. In addition to ${ }^{226} \mathrm{Ra}$ activities, ${ }^{230} \mathrm{Th}$ must be measured in the same barite to determine how much of the ${ }^{226} \mathrm{Ra}$ is supported.

3 . If the excess ${ }^{226} \mathrm{Ra} / \mathrm{Ba}$ ratios indicate young ages ( $<1000$ years), the ${ }^{232} \mathrm{Th}$ activity of the sample should be determined to correct ${ }^{228} \mathrm{Ra}$ activities so that excess $\left({ }^{228} \mathrm{Ra} /{ }^{226} \mathrm{Ra}\right)$ activity ratios can be determined. If the ${ }^{232} \mathrm{Th}$ activity is small, an approximate age could be determined from the ${ }^{228} \mathrm{Ra}{ }^{226} \mathrm{Ra}$ activity ratio.

4. Unless the ${ }^{232} \mathrm{Th}$ activities of these barites are extremely low, the ${ }^{228} \mathrm{Th} /{ }^{228} \mathrm{Ra}$ activity ratio will probably not be a reliable dating tool.

Because this was a preliminary investigation without any funding, we were unable to proceed with these labor-intensive analyses.

\section{REFERENCES ${ }^{*}$}

Moore, W.S., and Stakes, D., 1990. Ages of barite-sulfide chimneys from the Mariana Trough. Earth Planet. Sci. Lett., 100:265-274.

Phillips, G.W., and Marlow, K.W., 1976. Automatic analysis of gamma-ray spectra from germanium detectors. Nucl. Instrum. Methods. Phys. Res., 137:525-536.

Stakes, D., and Moore, W.S., 1991. Evolution of hydrothermal activity on the Juan de Fuca Ridge: observations, mineral ages, and Ra isotope ratios. J. Geophys. Res., 96:21739-21752.

\footnotetext{
-Abbreviations for names of organizations and publications in ODP reference lists follow the style given in Chemical Abstracts Service Source Index (published by American Chemical Society).
}

Date of initial receipt: 15 April 1993

Date of acceptance: 1 September 1993

Ms 139SR-223 
Table 1. Preliminary gamma-ray analyses of samples from Leg 139.

\begin{tabular}{|c|c|c|c|c|c|c|c|c|}
\hline $\begin{array}{l}\text { Sample } \\
\text { Leg } 139\end{array}$ & $\begin{array}{l}\text { Depth } \\
(\mathrm{cm})\end{array}$ & $\begin{array}{l}\text { Weight } \\
\text { (gm) }\end{array}$ & $\begin{array}{c}\mathrm{Ra}-226 \\
\text { (dpm) }\end{array}$ & $\begin{array}{c}\mathrm{Ra}-228 \\
(\mathrm{dpm})\end{array}$ & $\begin{array}{c}\text { Th-228 } \\
\text { (dpm) }\end{array}$ & $\begin{array}{c}{ }^{228} \mathrm{Ra} /{ }^{226} \mathrm{Ra} \\
\text { (A. R.) }\end{array}$ & $\begin{array}{c}{ }^{228} \mathrm{Th} /{ }^{228} \mathrm{Ra} \\
\text { (A. R.) }\end{array}$ & $\begin{array}{c}{ }^{226} \mathrm{Ra} \\
\text { (dpm/gm) }\end{array}$ \\
\hline $855 \mathrm{C}-010 \mathrm{X}-\mathrm{CC}$ & 13 & 30.8 & 22.9 & 20.7 & 23.5 & 0.90 & 1.14 & 0.74 \\
\hline $856 \mathrm{~A}-014 \mathrm{X}-\mathrm{CC}$ & 35 & 20.4 & 2.0 & $<$ det & 1.1 & & & 0.10 \\
\hline $\begin{array}{l}856 \mathrm{~B}-4 \mathrm{H}-3 \\
856 \mathrm{~B}-15 \mathrm{X}-\mathrm{CC}\end{array}$ & $\begin{array}{r}34 \\
4\end{array}$ & $\begin{array}{r}6.8 \\
17.1\end{array}$ & $\begin{array}{r}<\text { det } \\
9.0\end{array}$ & $\begin{array}{r}<\text { det } \\
8.2\end{array}$ & $\begin{array}{l}2.0 \\
9.0\end{array}$ & 0.91 & 1.11 & 0.53 \\
\hline $\begin{array}{l}856 \mathrm{D}-1 \mathrm{H}-1 \\
856 \mathrm{D}-1 \mathrm{H}-3 \\
856 \mathrm{D}-1 \mathrm{H}-4 \\
856 \mathrm{D}-1 \mathrm{H}-5 \\
856 \mathrm{D}-1 \mathrm{H}-5 \\
856 \mathrm{D}-1 \mathrm{H}-7\end{array}$ & $\begin{array}{r}129 \\
115 \\
33 \\
8 \\
8 \\
117 \\
73\end{array}$ & $\begin{array}{r}21.8 \\
23.1 \\
21.8 \\
9.7 \\
17.1 \\
24.1 \\
\end{array}$ & $\begin{array}{r}24.8 \\
10.3 \\
5.2 \\
<\text { det } \\
5.6 \\
114.0\end{array}$ & $\begin{array}{r}3.7 \\
0.0 \\
0.0 \\
1.9 \\
4.7 \\
<\text { det }\end{array}$ & $\begin{array}{l}0.8 \\
0.2 \\
0.0 \\
0.3 \\
0.1 \\
0.2\end{array}$ & $\begin{array}{l}0.15 \\
0.00 \\
0.00\end{array}$ & 0.22 & $\begin{array}{l}1.14 \\
0.45 \\
0.24\end{array}$ \\
\hline $\begin{array}{l}856 \mathrm{G}-3 \mathrm{R}-1 \\
856 \mathrm{G}-6 \mathrm{R}-1 \\
856 \mathrm{G}-7 \mathrm{R}-2 \\
856 \mathrm{G}-7 \mathrm{R}-3\end{array}$ & $\begin{array}{r}6 \\
46 \\
145 \\
10\end{array}$ & $\begin{array}{l}24.9 \\
23.9 \\
46.6 \\
47.1\end{array}$ & $\begin{array}{r}4.3 \\
28.4 \\
20.4 \\
14.8\end{array}$ & $\begin{array}{r}5.5 \\
1.3 \\
<\text { det } \\
<\text { det }\end{array}$ & $\begin{array}{r}0.4 \\
0.6 \\
0.8 \\
<\text { det }\end{array}$ & $\begin{array}{l}1.28 \\
0.05\end{array}$ & $\begin{array}{l}0.07 \\
0.43\end{array}$ & $\begin{array}{l}0.17 \\
1.19 \\
0.44 \\
0.31\end{array}$ \\
\hline $856 \mathrm{H}-1 \mathrm{R}-1$ & 34 & 25.0 & 79.6 & 0.9 & 2.2 & 0.01 & & 3.18 \\
\hline $857 C-68 R-1$ & 139 & 34.5 & 3.7 & 2.1 & 2.6 & 0.57 & 1.24 & 0.11 \\
\hline $\begin{array}{l}858 \mathrm{~A}-00 \mathrm{X}-5 \\
858 \mathrm{~A}-00 \mathrm{X}-3 \\
858 \mathrm{~A}-014 \mathrm{X}-1\end{array}$ & $\begin{array}{l}56 \\
41 \\
28\end{array}$ & $\begin{array}{r}16.6 \\
8.8 \\
14.4\end{array}$ & $\begin{array}{r}13.2 \\
6.0 \\
5.9\end{array}$ & $\begin{array}{r}16.8 \\
14.8 \\
8.9\end{array}$ & $\begin{array}{r}15.0 \\
7.5 \\
8.7\end{array}$ & $\begin{array}{l}1.27 \\
2.45 \\
1.50\end{array}$ & $\begin{array}{l}0.89 \\
0.51 \\
0.98\end{array}$ & $\begin{array}{l}0.80 \\
0.69 \\
0.41\end{array}$ \\
\hline $\begin{array}{l}858 \mathrm{~B}-5 \mathrm{H}-2 \\
858 \mathrm{~B}-2 \mathrm{H}-3 \\
858 \mathrm{~B}-2 \mathrm{H}-4\end{array}$ & $\begin{array}{r}13 \\
133 \\
21\end{array}$ & $\begin{array}{l}42.8 \\
14.0 \\
21.0\end{array}$ & $\begin{array}{l}91.7 \\
12.4 \\
11.5\end{array}$ & $\begin{array}{r}11.5 \\
9.0 \\
<\text { det }\end{array}$ & $\begin{array}{r}11.1 \\
1.7 \\
0.9\end{array}$ & $\begin{array}{l}0.13 \\
0.73\end{array}$ & $\begin{array}{l}0.97 \\
0.19\end{array}$ & $\begin{array}{l}2.14 \\
0.89 \\
0.55\end{array}$ \\
\hline $\begin{array}{l}858 \mathrm{C}-5 \mathrm{H}-1 \\
858 \mathrm{C}-5 \mathrm{H}-2 \\
858 \mathrm{C}-6 \mathrm{H}-3 \\
858 \mathrm{C}-6 \mathrm{H}-5 \\
858 \mathrm{C}-6 \mathrm{H}-1 \\
858 \mathrm{C}-11 \mathrm{X}-\mathrm{CC} \\
858 \mathrm{C}-12 \mathrm{X}-2 \\
858 \mathrm{C}-12 \mathrm{X}-\mathrm{CC}\end{array}$ & $\begin{array}{r}141 \\
28 \\
84 \\
83 \\
129 \\
32 \\
36 \\
23\end{array}$ & $\begin{array}{l}42.2 \\
38.0 \\
20.8 \\
11.1 \\
12.3 \\
27.4 \\
23.2 \\
34.6\end{array}$ & $\begin{array}{r}34.4 \\
27.3 \\
9.4 \\
5.8 \\
8.7 \\
8.7 \\
15.2 \\
9.1 \\
15.5\end{array}$ & $\begin{array}{r}44.6 \\
36.8 \\
10.0 \\
4.0 \\
19.0 \\
19.9 \\
19.0 \\
27.0\end{array}$ & $\begin{array}{r}42.3 \\
32.4 \\
8.8 \\
5.1 \\
12.4 \\
21.4 \\
12.6 \\
20.6\end{array}$ & $\begin{array}{l}1.30 \\
1.35 \\
1.06 \\
0.70 \\
2.19 \\
1.31 \\
2.09 \\
1.74\end{array}$ & $\begin{array}{l}0.95 \\
0.88 \\
0.88 \\
1.28 \\
0.65 \\
1.08 \\
0.66 \\
0.76\end{array}$ & $\begin{array}{l}0.82 \\
0.72 \\
0.45 \\
0.52 \\
0.71 \\
0.55 \\
0.39 \\
0.45\end{array}$ \\
\hline $858 \mathrm{~F}-025 \mathrm{R}-1$ & 101 & 25.0 & 6.1 & $<$ det & 1.9 & & & 0.24 \\
\hline
\end{tabular}

Notes: $<$ det $=$ below detection . 